\title{
Flow Visualisation by Condensing Steam - an Unusual Method Applied to Development of a Low Reynolds Number Fluidic Selector Valve
}

\author{
J. R. Tippetts, V. Tesař
}

A visualization method so far not mentioned in the literature has been recently developed by the authors as a useful validation supplement to numerical flowfield computations in the design of microfluidic devices. The method is based upon water vapour condensation on device channel walls. It is extremely easy to set up with minimum expense - and yet it is very reliable. As an application example, the paper shows the method used in study of properties of a microfluidic valve intended for switching gaseous sample flows in a microfluidic selector sampling unit. A scaled-up model of the valve was built, as usual, in transparent acrylic material, making possible observation and photo-recording of the deposition and subsequent drying of the condensed droplets. The scaling-up slowed down the time scale enough for investigating the transition processes which takes place as the flow in the valve is switched on and off.

Keywords: flow visualization, condensing steam, microfluidics, valves.

\section{Introduction}

Visualization of fluid flow [3], [4] is an important research tool for obtaining information about mechanisms of flows and also a validation and confirmation tool for developing hydraulic and pneumatic devices or - to use a more general term - fluidic devices [5]. In internal flow problems, visualisation usually requires making a model of the device with transparent walls, but this is rarely a problem since the commonly used transparent acrylic material (polymethylmetacrylate) is cheap, sufficiently soft for convenient and fast machining, and stiff enough to resist the acting mechanical forces. The main problem is that the common fluids used in laboratory investigations - water and air - are transparent and require an additional facility to generate observable effects. For many purposes, this causes inconvenient complications, and there is a general demand for visualisation facilities which would be simple and inexpensive. The problem is less severe in the case of water, which may be relatively simply coloured using a dye. For air flows, an analogous addition of smoke has been tried, but the smoke is inconvenient to generate, usually not well observable in narrow channels (where there cannot be enough smoke for significant light extinction). Also, the "smoke" - actually droplets of decomposition products - tends to condense in the channels, generating a non-transparent layer on the walls which obstructs the observation and requires repeated cleaning of the model.

A new method described in the present paper is used for studying the air flow in the channels of acrylic models. It uses condensation of water vapour on the channel walls. The patterns of the regions with consensed water droplets are easily observable. Since the vapour deposition depends on local flow velocity (and local shear stress on the wall), and the droplets tend also to be removed from a high-velocity location (either because they evaporate or because they are moved by the shear stress), the condensation pattern provides a useful picture of the conditions inside the channel. The method is extremely simple and cheap. Somewhat surprisingly, there does not seem to be any description of this method in authoritative monographs (e.g. [3, 4]) on flow visualization methods.

\section{The task}

Present authors find flow visualization indispensable for their current development of no-moving-part fluidic devices, in particular flow control valves. Despite the convenience of CFD numerical flowfield solutions as a tool for designing the internal geometry of the valve, experience shows that it cannot be relied upon without verification experiments. This may sound rather surprising in the context of microfluidics, with devices usually operated at low Reynolds numbers. Indeed, microfluidic flows are usually laminar and the numerical solution is thus spared the complexities and intricacy of turbulence modeling, the usual source of unreliability and troubles. This, however, is not valid universally - there are microfluidic devices with fully turbulent flows. Moreover, even at moderate and low Reynolds numbers the character of the flow may be complicated by vortices which the computations - especially the usual steady-state computations - may not always handle properly.

The developed valves are based on the flow diverting principle. The supplied flow forms a jet deflected by the action of the control flow. In particular, the described novel visualisation method was developed and used in verification experiments with pressure-driven valves intended for applications in fluid sample selectors $[2,8]$ for delivering samples to chemical composition analyser. The need for sample purity results in a very special requirement - the generation of a jet pumping effect by the control flow. The pumping reverses the flow in the downstream cavities and this removes any remains of a sample that may linger in the conduits and other volumes connecting the valve with the composition analyzer further downstream. Achieving a sufficiently strong entrainment into the jet called for the control jet Reynolds number to be much higher than is usual in microfluidics, reaching values of the order of $\operatorname{Re}=1000$. This is still mostly within the lami- 
nar regime, but the flows become dominated by quite strong vortices, actually welcome for generating an effective entrainment effect in the jet.

Apart from the desirable vortices entraining fluid in the jet, the geometry gave rise to various other vortices, more or less standing inside various corners. The problem with CFD solution unreliability was traced down to the software's improper handling of these vortices (Tesař et al., 2004 - [2, 12]). Even the most sophisticated software versions - large-eddy simulations - tend to compute the vortices as steady, while in reality (as documented by the flow visualizations) they are shed and carried away with the flow. Other vortices are then formed anew in their place. The energy for their formation is extracted from the main flow. As a result, this was found to lead to seriously overpredicted diffuser effects and underpredicted losses [12].

A disappointing experience with the initial CFD results led to an altered design procedure in which the computations are validated by laboratory tests using large scale acrylic (Perspex) models. One attractive feature of the scaled-up models is the significantly slower, easier to investigate switching frequency in the model. This is due to the fact that the scaling factor of the switching is the Stokes number

$$
\mathrm{Sk}=\frac{f \cdot d^{2}}{v}
$$

When increasing the size $d$ of the model - as the authors did - five times relative to the actual microvalve, hydrodynamic similarity (= equal Sk) requires switching at 25-times lower frequency $f$ if the kinematic viscosity $v$ remains the same. As a matter of fact, the viscosity of our model fluid is lower. The valve is designed for switching "syngas" - synthesis gas containing a substantial proportion of a high-viscosity $\mathrm{H}_{2}$ component, at a high temperature $(400 \mathrm{~K})$ - while our model experiments are run with much cooler air, having roughly 3-times lower viscosity. As a result, the switching frequency for the same Stokes number in the model is about 75-times lower. This makes the switching processes in the model easily observable - and recordable by a standard camcorder.

Requirements concerning the resolution power of the flow visualization method in these tests are certainly undemanding. We need not study fine details of the flowfield. The interest is mainly in detecting into which channel the sample flow passes in response to the admission of the control flow. Standard, expensive gas flow visualization methods, aiming at revealing the fine points of the flowfield, are unnecessary. After all, on a 5:1 scale the model channel widths are still rather small, of the order of millimetres, which is certainly not enough for any elaborate study of the flow details. On the other hand, with the visualization having in our case a more or less supplementary role, we need the method to be inexpensive, readily available and reliable.

The standard gas flow visualization, which we could have used but decided not to, is the well known so called "smoke" method (e.g. Merzkirch 1974 [3]). The gas motion is made visible by particles of "oil smoke" carried with the flow. In fact the particles are formed by condensing oil vapours. For our purpose, we found several unpleasant disadvantages. Setting up an efficient "burning" (in fact mainly evaporation) of the very small properly dosed oil flow for reasonable constancy of vapour concentration is by no means easy. Good results certainly require a professionally built facility, which is not exactly cheap. Another problem is the fouling of the model channels - sedimentation of the condensed particles on the walls - producing a difficult to remove layer that gradually obscures the view into the channel.

What we needed was a simple, cheap and easy to set-up method, leaving no permanent deposits on the wall. Similar requirements may arise in many other situations, where our experience may be found useful.

\section{Condensing steam method}

The inspiration for the development of the new method came from observations of our colleagues who tested the behaviour of the newly delivered model by blowing into its terminal inlets with their mouths. Though extremely crude, this method enabled some observation of the switching action, because the humidity contained in human breath was seen to condense on the walls of the model channels through which the breath passed, revealing a clearly visible gas flowpath inside the model.

A more permanent source of moist air was built according to Fig. 1. The air flow rate is measured upstream, where the air is still dry. The moisture content is then increased by bubbling the air through water in a container, which is easily improvised from a plastic bottle for soft drinks. We used a standard PET bottle of 1.5 litre volume. Of course, the bottle has to be closed tightly to prevent leakage of the air. For the air inflow and outflow, the bottle plug was adapted by drilling through two holes with two different lengths of plastic tubing inserted and glued into the plug. One of them reaches deep below the water level, while the end of the other one remains above the water.

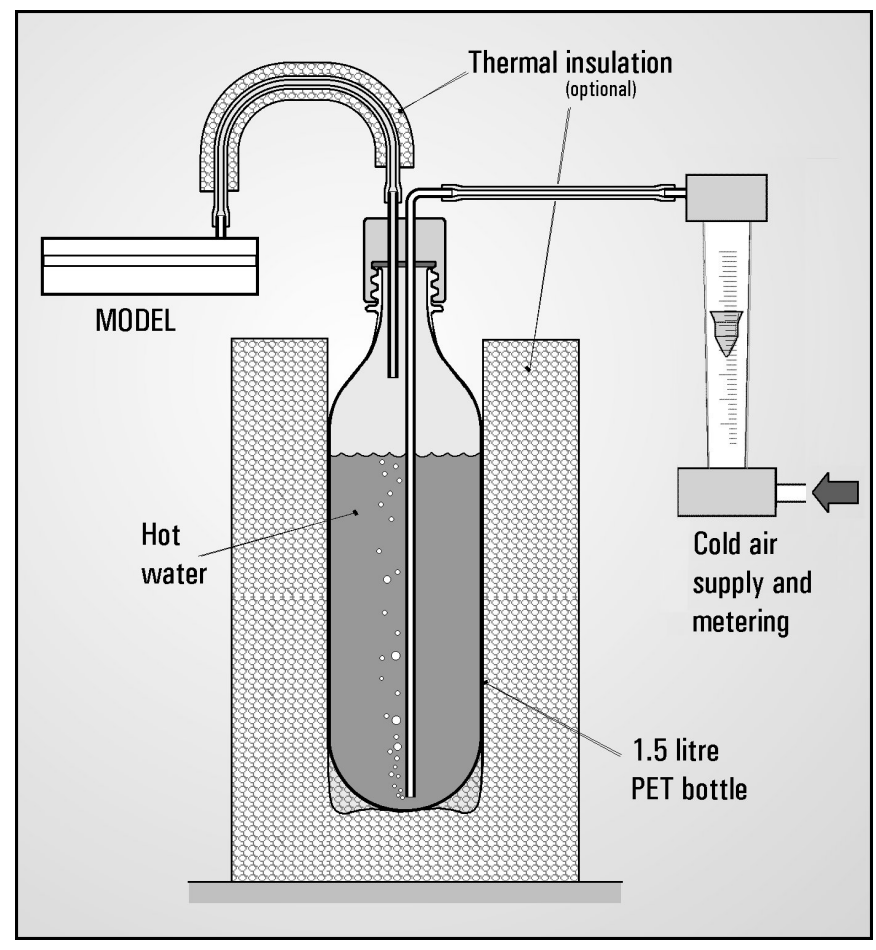

Fig. 1: Schematic arrangement of the humid air source. The only component needed is any suitable bottle filled with hot water. The authors use an empty Coca Cola bottle, filled with water heated in the office tea kettle. 
The saturation with moisture was found to improve significantly if the water temperature was increased. It is easy to fill the bottle with hot and indeed boiling water. We warmed it simply in the office tea kettle. For protracted experiments the bottle was provided with polystyrene thermal insulation (using the polystyrene inserts from the boxes in which computers are delivered). Also advisable (though not seen in our setup shown in Fig. 6), is then to provide a similar thermal insulating layer for the feed pipe leading to the model (as shown in Fig. 1). It is useful to keep this pipe short (the length of our $5 \mathrm{~mm}$ i.d. pipe was $100 \mathrm{~mm}$ ). Even with the complicating feature of heated water, anyone can improvise the moist air source according to Fig. 1 a few minutes. The cost is virtually zero. The advantage of water condensation is that the generated droplets are easily removed from the surfaces after the visualization experiment by simply blowing dry air through the model. The water is in effect distilled and leaves no fouling residua.

Although the water vapour condensation in the air flow inside the cavities is also observable, we relied mainly on the clearly visible layer of water droplets created by condensation on the cool walls of the model channels. The layer being white, it is useful to improve the observation by placing a contrasting black surface behind the model (Fig. 6). The model may be cooled to enhance the condensation - we tested cooling it by tap water or by air flow from a small blower - but this is not strictly necessary. We used pressure cylinders as the source of our control flow and found particularly well observable condensation provided by a collision of the warm moist air flow from the valve supply nozzle with a control flow, which is cooled by the expansion from the cylinder prior to the entry into the model. The condensed water droplets on the surfaces are very small and are removed quite fast if exposed to dry control air. The changes in the optical properties of the condensed layer - the droplet deposition as well as removal by drying - are fast enough for investigations of the switching processes in the valve.

Of course, in the variant utilizing condensation on the walls, the method is particularly suitable for investigating fluidic circuits behaviour, with emphasis on the switching processes and on following the flowpaths. It is obviously less useful for a study of the internal flowfield inside the channel, though it may indicate some interesting facts, such as the boundaries between the moist and dry gas flows if they share the same channel. It can also provide some information about the orientation and magnitude of the wall shear stress.

\section{Application of the method}

\subsection{Object of investigations}

Although of quite wide applicability, the present method was developed and tested in studies of a microfluidic valve. The valve is a successor to several earlier designs (Tesař $[7,8]$; Tesař et al., [6]). It is intended to form a part of a 16-channel fluidic flow selector sampling unit to be used for catalyst testing (Low et al., 2001 [1]; Wilkin et al., 2002 [10]). The details of its layout and its properties are not discussed here as they are available elsewhere (Tesař et al., 2004 [2]).

The valve is of planar arrangement, with relatively shallow cavities (the cavity contours are shown in Fig. 4) of everywhere constant depth. It is operated in two principal states: "OPEN" and "CLOSED". In the sampling unit, all but one of the valves are in their "CLOSED" state while the only remaining one is "OPEN", permitting the flow of the corresponding one fluid sample to a composition analyzer.

In the "OPEN" state, shown in the schematic representation Fig. 2, there is no control flow. The sample flow passes through the valve from the supply terminal $S$ to the output terminal Y. The hydrodynamic conditions in the valve are determined by the very small sample supply flow rate, rendering the Reynolds numbers very low - its value is only around $\operatorname{Re}=88$, evaluated from the conditions in the supply nozzle. This is too small for efficient driving of the sample flow into the connected load (analyzer). Instead, the sample is driven there by the applied constant pressure difference $\Delta \mathrm{P}_{\mathrm{YV}}$. This is applied between the mutually interconnected vents $V$ and the output terminals $\mathrm{Y}$ of all valves forming the sampling unit. To prevent contamination of the sample by the fluid mixture from the vent, the pressure is adjusted so as to generate a small spillover "guard" flow into the vent $\mathrm{V}$.

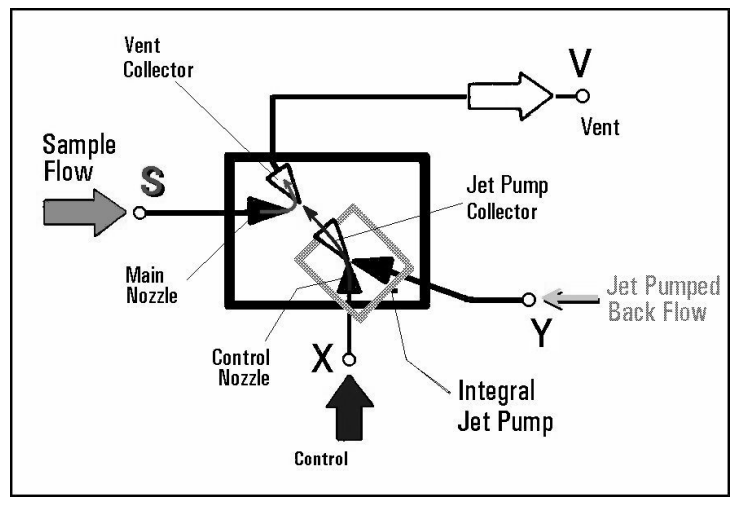

Fig. 2: Schematic diagram of the valve. Black triangles: nozzles, white triangles: collectors. In the OPEN state shown here the sample flow from supply terminal $S$ passes into output Y. A small percentage is spilled over as the protective "guard" flow into vent V.

The "CLOSED" state, represented schematically in Fig. 3, is brought about by the admission of the control flow to the control terminal X. The sample flow is not actually turned down. As shown in Fig. 3, it is instead prevented from enter-

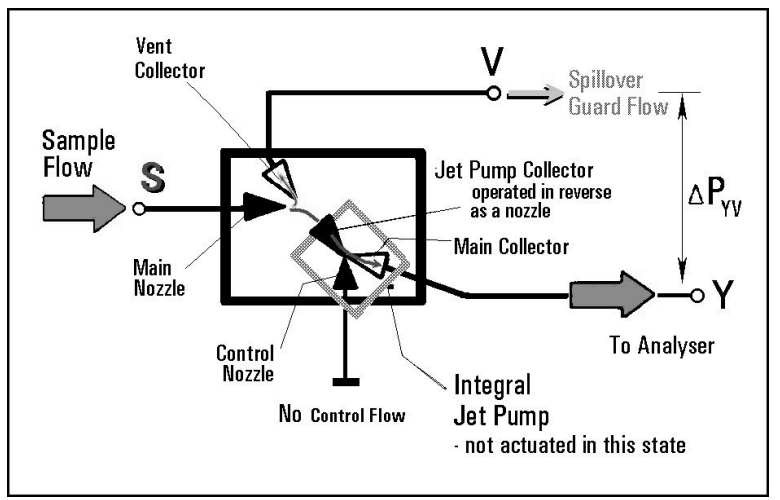

Fig. 3: Schematic diagram of the valve in the CLOSED state. To clean the cavities downstream from Y, the powerful control flow from $\mathrm{X}$ generates a reverse output flow in the jet pump part of the valve. 
ing the valve output $\mathrm{Y}$ by being diverted into the vent $\mathrm{V}$. The control flow actually meets the supply flow already slowed down in the diffuser (note the white triangle symbol of the diffuser in Fig. 3) of the jet pump collector so that the diverting effect is not so much by the jet momentum interactions used in the large-scale fluidic devices (e.g., Tesař, 1998) as it is due to fact that the entrance into the output channel is obstructed by the control fluid presence. The control jet leaving the control nozzle is here rather extraordinarily powerful. Its Reynolds number is as high as $\operatorname{Re}=1000$, a value out of proportion in usual microfluidics, but required here for formation of vortical motions in the control jet. These produce a quite effective entrainment of the fluid from the output channel, this generating a return flow in the output terminal $\mathrm{Y}$, required for cleaning the downstream cavities between the valve and the analyzer. It is in this "CLOSED" state - and the transitions between the "OPEN" and "CLOSED" regimes that the problems with CFD unreliability occur.

\subsection{Visualisation experiments}

The enigmatic disagreements between the computations and the experimental data [12] were investigated using 5:1 scaled up models of the valves. The interaction cavities, as shown in Fig. 4, were made by laser cutting in a Perspex plate.

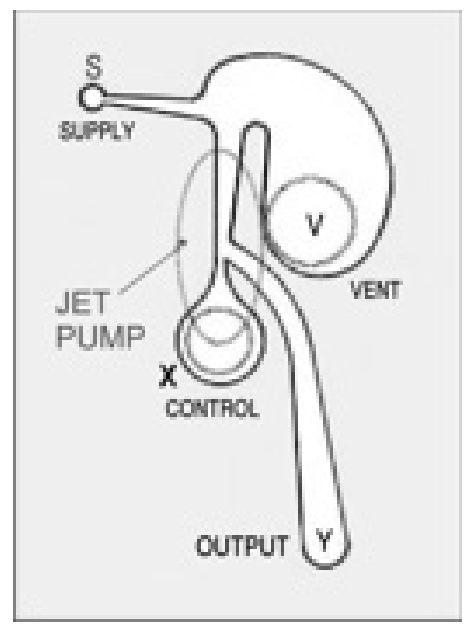

Fig. 4: Contours of the valve cavities. The layout is planar and is dominated by what is in effect an incorporated jet pump, driven by the control flow.

The plate is clamped between the top and bottom cover plates. The latter are quite thick (they may be seen in Fig. 6) and hold the tube connection ferrules of the four terminals of the valve. Of them, three terminals $(\mathrm{S}, \mathrm{X}$, and $\mathrm{Y})$ are in the top plate (Fig. 6), unfortunately somewhat obstructing the observation in Figs. 5 and 7 (the valves were viewed from the top during the experimental runs). It should be said that the models were intended for working purposes, to obtain information, not to generate nice visualization pictures. Nevertheless, despite the somewhat cluttered appearance of the pictures taken during the runs, the essential information - the presence or absence of the gas flow in the individual parts of the valve - is clearly recognizable.

By way of example, Fig. 7 presents a photograph of the model in the "OPEN" state. The humid air entering from the supply terminal S leaves well observable traces on its way into the output terminal Y. It is not only possible to trace the flow

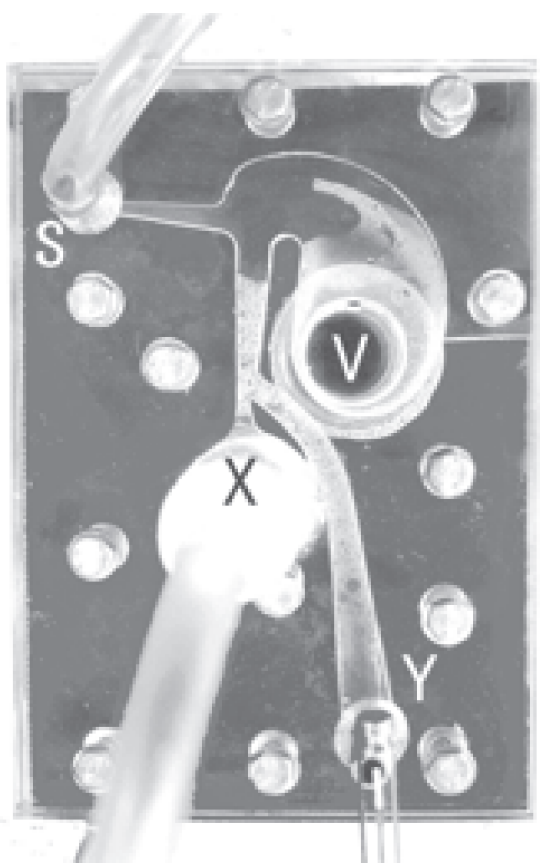

Fig. 5: Photograph of the scaled-up acrylic model of the valve as used in the laboratory flow visualisation experiments using the steam condensation method - some condensation is visible in the channels

in the channels, but in the large cavity area downstream from the supply channel it is also possible to see a part of the supplied gas spilling over into the vent V. This shows the desirable generation of the small spillover "guard" flow, which eliminates the possibility of the uncontrolled fluid mixture in the common vents getting into contact with the sample. Though a back flow from the vent $\mathrm{V}$ is not very likely, its existence cannot be ruled out in the absence of any mechanical separation (which would rule out such a possibility in mechanically closing valves). The "guard" flow represents a sacrifice of a part of the sample and should not therefore be higher than strictly necessary. This is adjusted by proper

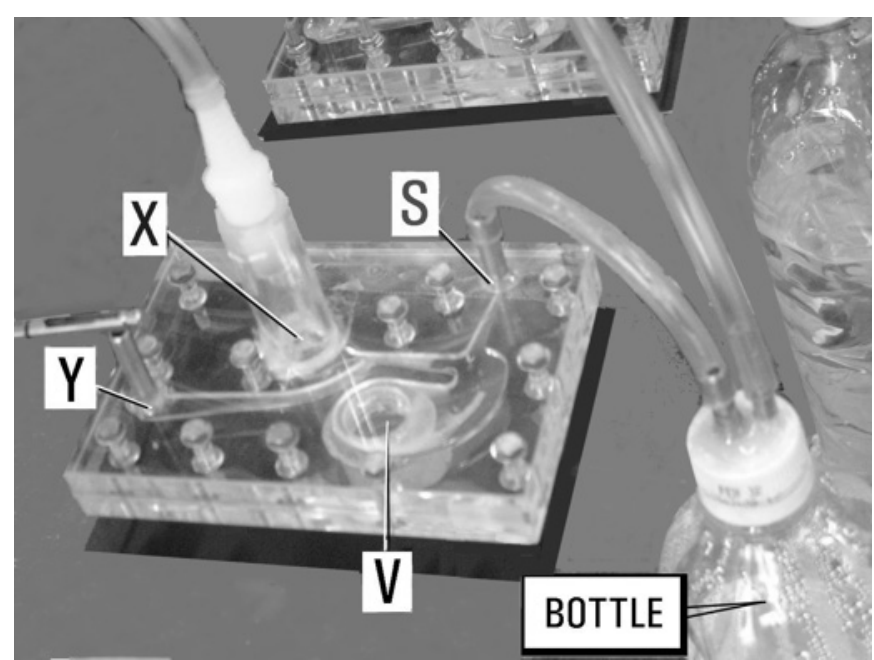

Fig. 6: Oblique view of the acrylic model, with the top of the bottle filled with hot-water seen in the foreground. Partly visible in the background are another model and its bottle - the tests were performed with the whole multi-valve selector circuit. 


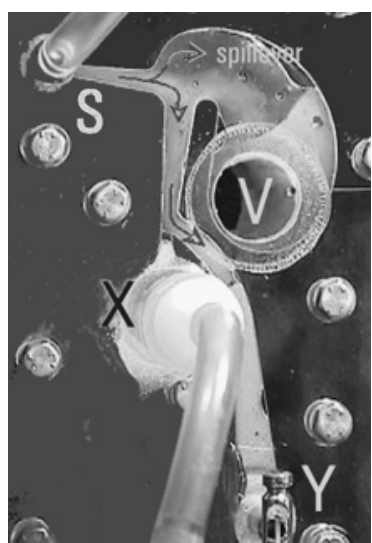

Fig. 7: Visualised flow in the model valve in its OPEN state. Clearly recognisable due to the condensation is the spillover flow into vent V. Its magnitude can be estimated.

choice of the applied driving pressure difference DPYV. Observing the amount of the flow sacrificed in situations like that shown in Fig. 7 is a useful aid for the adjustment.

Fig. 8 presents a sequence of the flow visualization photographs taken during the transition from the "OPEN" state (no control flow) into the "CLOSED" state. It should be noted how the condensation in the path of the control flow in the jet pump part of the valve gradually clears away. This indicates the inaccessibility of the path to the valve output $\mathrm{Y}$ for the humid supplied gas once the control flow is switched on. After a mere 4 seconds, the switching into the "CLOSED" state is completed. This represents a very short transition in the actual valve in view of the Stokes number similarity Eq. (1) above.

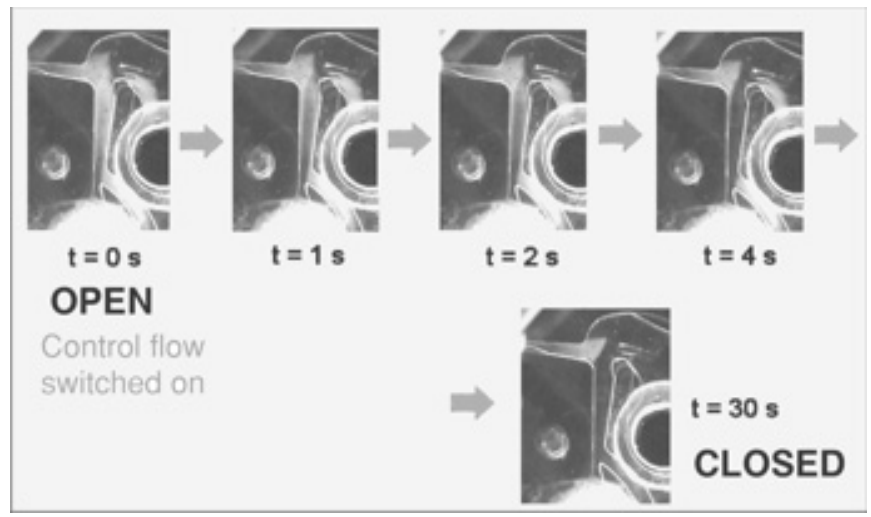

Fig. 8: Sequence of video records showing the transition from the OPEN state into the CLOSED state by the action of the control flow. The control air removes the condensed water droplets.

An effect which deserves pointing out is the much slower but demonstrably present gradual clearing of the condensation in the output channel, between the jet pump part of the valve and the output terminal $\mathrm{Y}$. This manifests the presence of the reverse flow, cf. Fig. 3, generated due to jet pumping. This is an important feature required for maintaining sample purity. The fact that this reverse flow is smaller - so that the condensation removal of took 30 seconds in the scaled-up experiment - is actually quite desirable. The generation of the reverse flow takes place in the "CLOSED" state and since in the sampling unit there is a large number of the valves simultaneously in this "CLOSED" state, the combined loss of the sample resultant from all protective reverse flows may easily represent a substantial proportion of the available sample. We must keep it small and this is achieved by generating only rather weak jet pumping - well within the capability of the essentially laminar control jet.

\section{Conclusions}

In the design of fluidic devices similar to our microfluidic valve, flow visualization is a useful and in fact indispensable validation tool accompanying the numerical flowfield computations. Because of the auxiliary nature of the visualization and the lack of interest in revealing details of the flow, the main requirement on the flow visualization methods for this purpose is simplicity and low cost. The steam condensation method, described in this paper, fulfills the requirement almost ideally.

\section{Acknowledgment}

The authors gratefully acknowledge financial support from iAc - Institute of Applied Catalysis, United Kingdom.

\section{References}

[1] Low, Y. Y., Tesař, V., Tippetts, J. R., Allen, R. W. K., Pitt, M.: "Mutlichannel Catalyst Testing Reactor with Microfluidic Flow Control." $3{ }^{\text {rd }}$ European Congress of Chemical Engineering, Nuremberg, Germany, 2001.

[2] Tesař, V., Tippetts, J. R., Low, Y., Y., Allen, R. W. K.: "Development of a Microfluidic Unit for Sequencing Fluid Samples for Composition Analysis." Chemical Engineering Research and Design, Vol. 82 (A6) (2004), p. 708-718, Transactions of the Institution of Chemical Engineers, U.K., Part A, June 2004.

[3] Merzkirch, W.: Flow Visualization. Academic Press, New York, 1974.

[4] Ǩezníček, R.: Visualisace proudění. (Flow Visualization, in Czech), ACADEMIA publishing house of ČSAV, Praha, 1972.

[5] Tesař, V.: "Valvole fluidiche senza parti mobili." (No-Moving-Part Fluidic Valves, in Italian), Oleodinamica - pneumatica, revista delle applicazioni fluidodinamiche e controllo del sistemi, Vol. 39 (1998), No. 3, ISSN 1 122-5017 , p. 216.

[6] Tesař, V., Low, Y. Y., Allen, R. W. K., Tippetts, J. R.: "Microfluidics for MEMS - Microfluidic Valve", 2001 PICAST IV - Proceedings of the $4^{\text {th }}$ Pacific International Conference on Aerospace Science and Technology, Kaoshiung, Taiwan, p. 301-306, Publ. by: National Cheng Kung University, Tainan, Taiwan, 2001.

[7] Tesař, V.: "Microfluidic Valves for Flow Control at Low Reynolds Numbers", Journal of Visualisation, Vol. 4 (2001), No. 1, p. 51-60, Tokyo.

[8] Tesař, V.: "Sampling by Fluidics and Microfluidics", Acta Polytechnica - Journal of Advanced Engineering, Vol. 42 (2002), No. 2, Prague, p. 41-49. 
[9] Tesař, V.: „Subdynamické asymptotické chování mikrofluidického ventilu" (Subdynamic Asymptotic Behaviour of a Microfluidic Valve, in Czech), Automatizace, ISSN 0005-125X, Vol. 45 (2002), No. 12, p. 766-770, Prague.

[10] Wilkin, O. M., Allen, R. W. K., Mailtlis, P. M., Tippetts, J. R., Tesař, V., Turner, M. L., Haynes, A., Pitt, M. J., Low, Y. Y., Sowerby, B.: „High Throughput Testing of Catalysts for the Hydrogenation of Carbon Monoxide to Ethanol.“ In: Derouanne E. G. et al.: „Principles and Methods for Accelerated Catalyst Design and Testing", p. 299-303, ISBN 1402007205, Kluwer Acad. Publishers, Netherlands, 2002.

[11] Tesař, V.: "Microfluidic Turn-down Valve." Journal of Visualisation, Vol. 5 (2002), No. 3, p. 301-307, Tokyo.

[12] Tesař, V., Tippetts, J. R., Allen, R. W. K.: "Failure of Steady CDF Solutions Caused by Vortex Shedding." Developments in Machinery Design and Control, Vol. 3, ed.
K. Peszynski, ISBN 83-89334-85-2, p. 87-94, Poland, 2004.

Dr. J. R. Tippetts, Ph.D.

e-mail: j.tippetts@sheffield.ac.uk

University of Sheffield

Mappin Street S1 3JD

Sheffield, UK

Prof. Ing. Václav Tesař, CSc.

e-mail:v.tesar@sheffield.ac.uk

University of Sheffield

Mappin Street S1 3JD

Sheffield, UK

AV ČR

Dolejškova 5

18200 Praha 8, Czech Republic 